\title{
Valoración de las estrategias adoptadas por docentes en la enseñanza de la ciencia desde la perspectiva de los estudiantes de educación básica*
}

Assessment of the strategies adopted by teachers in the teaching of science from the perspective of basic education students

Avaliação das estratégias adotadas pelos professores no ensino de ciências na perspectiva de alunos da educação básica

Recibido el 12 de mayo de 2017. Aceptado el 1 de febrero de 2018

Para citar este artículo:

Veloza Rincón, Ramiro Alejandro

y Hernández Suárez, César

Augusto (diciembre, 2018).

Valoración de las estrategias

adoptadas por docentes en la

enseñanza de la ciencia desde la

perspectiva de los estudiantes

de educación básica. Ánfora,

25(45), 43-69. DOl: https://doi.

org/10.30854/anf.v25.45.2018.

XXX Universidad Autónoma de

Manizales. ISSN 0121-6538.

\author{
Ramiro Alejandro Veloza Rincón** \\ Colombia \\ César Augusto Hernández Suárez*** \\ Colombia
}

\section{Resumen}

Objetivos: caracterizar y evaluar el nivel de desarrollo de las competencias pedagógicas mediante las estrategias adoptadas por docentes de Educación Básica en la enseñanza de la ciencia desde la perspectiva de los estudiantes. Metodología: se aplicó un enfoque metodológico cuantitativo de naturaleza descriptiva y multifactorial. Los datos se obtuvieron entre los

\footnotetext{
* Este artículo se deriva del "Proyecto enjambre fortalecimiento de la cultura ciudadana, virtual, digital y democrática en ciencia, tecnología e innovación, a través de la investigación como estrategia pedagógica apoyada en las TIC en instituciones educativas de Norte de Santander" de la Gobernación Norte de Santander financiado por el Sistema General de Regalías. Proyecto iniciado en septiembre de 2015 y terminado en octubre de 2016.

** Magister en Prácticas Pedagógicas. Profesor de la Institución Educativa Santiago Apóstol, Santiago, Norte de Santander. Correo: alejoveloza@hotmail.com

*** Doctor en Formación en Ciencias de la Educación. Magister en Enseñanza de las Ciencias. Docente investigador de la Universidad Francisco de Paula Santander, Cúcuta, Norte de Santander. Director del Grupo de Investigación en Pedagogía y Practicas Pedagógicas (GIPEPP). Correo: cesaraugusto@ufps.edu. CO
} 
estudiantes de octavo y noveno grado de la Institución Educativa Santiago Apóstol, Municipio de Santiago, Norte de Santander, Colombia. Se utilizó una escala Likert diseñada para que los estudiantes describieran la práctica pedagógica de los docentes del área de Ciencias Naturales desde los tres aspectos que se evalúa en el desarrollo de la competencia pedagógica: la planeación curricular, desarrollo curricular y seguimiento y mejora (evaluación curricular). Resultados: las pruebas estadísticas muestran que los docentes desarrollan prácticas pedagógicas reflexivas que requieren de refuerzo en la Estrategia Pedagógica (IEP) y en las Tecnologías de la Información y las Comunicaciones (TIC) que permitan un desarrollo de competencias científicas más asertivas. Conclusiones: se identifican estrategias que promueven el desarrollo de competencias científicas en los estudiantes y se devela la utilización de recursos didácticos y prácticas de laboratorio que, sin embargo, no están articulados con las necesidades de los estudiantes.

Palabras clave: Práctica pedagógica; Enseñanza de la ciencia; Competencias.

\section{Abstract}

Objective: to characterize and assess the level of development in pedagogical competences through the strategies adopted by Basic Education teachers in the teaching of science from the students' perspective. Methodology: A quantitative approach was applied which was of a descriptive and multifactorial nature. The data was collected among the eighth and ninth grade students of the Santiago Apóstol Educational Institution, municipality of Santiago, Norte de Santander, Colombia. A Likert scale was used for students to describe the pedagogical practice of teachers in the area of Natural Sciences including the three aspects that are assessed in the development of pedagogical competence: curricular planning, curriculum development and curriculum assessment (monitoring and improvement). Results: the statistical tests show that teachers develop reflective pedagogical practices which require reinforcement in the Research Based Instructional Strategy (RBIS) and in Information and Communication Technologies (ICT) leading to the development of more assertive scientific competences. Conclusions: strategies were identified to foster the development of scientific competences in students and the use of didactic resources and laboratory practices, however, they are not articulated to the needs of students.

Keywords: Pedagogical practice; Teaching of science; Competences. 


\section{Resumo}

Objetivo: caracterizar e avaliar o nível de desenvolvimento das competências pedagógicas mediante as estratégias adotadas pelos professores de Educação Básica no ensino da ciência a partir da perspectiva dos alunos. Metodologia: foi aplicado um enfoque metodológico quantitativo de natureza descritiva e multifatorial. Os dados foram obtidos entre os alunos de oitava e nona série da Instituição Educativa Santiago Apóstol, Município de Santiago, Norte de Santander, Colômbia. Foi utilizada uma Escala Likert desenhada para que os estudantes descrevessem a prática pedagógica dos professores na área de Ciências Naturais a partir de três aspectos avaliados no desenvolvimento da competência pedagógica: planejamento de currículo, desenvolvimento de currículo e seguimento e melhoria (avaliação curricular). Resultados: as provas estatísticas mostram que os professores desenvolvem práticas pedagógicas reflexivas que precisam de um reforço na Estratégia Pedagógica (IEP) e nas Tecnologias da Informação e das Comunicações (TIC), reforços que vão levar a um desenvolvimento mais assertivo das competências científicas. Conclusões: identificamse estratégias que promovam o desenvolvimento de competências científicas nos estudantes e se evidencia o uso de recursos didáticos e práticas de laboratório que, no entanto, não estão coordenados com as necessidades dos estudantes.

Palavras-chave: Prática pedagógica; Ensino da ciência; Competências. 


\section{Introducción}

En los últimos años en Colombia se han generado políticas educativas para desarrollar planes de mejoramiento de la educación nacional que permitan su armonización con los retos y desafíos de la sociedad globalizada contemporánea. Entre estas políticas se encuentra la creación y consolidación de mecanismos de seguimiento y control sobre los resultados de las pruebas internas que se aplican a través del Instituto Colombiano para el Fomento de la Educación Superior (ICFES).

El Estado colombiano participa también en evaluaciones de carácter internacional, tales como las pruebas PISA (Programa Internacional de Evaluación de Estudiantes). PISA, como se conoce, es un proyecto que la Organización para la Cooperación y el Desarrollo Económico (OCDE) ha desarrollado desde finales de la década de 1990 con el objetivo de evaluar qué tan bien preparados están los estudiantes de 15 años para enfrentar los retos de la vida adulta (ICFES, 2013).

Los resultados entre el 2006 y 2009 de Colombia en las pruebas mencionadas, aunque con variaciones en función de las áreas de conocimiento, evidencian que los estudiantes del país tienen un rendimiento en general bajo. Para el 2012, el puntaje de Colombia fue inferior al obtenido por 61 países; en el caso de las ciencias, estuvo por detrás de 57 (ICFES, 2013). Ante estos resultados, se decidió la creación del Índice Sintético de Calidad, que busca que cada institución educativa se mida a sí misma en función de cuatro factores (progreso, desempeño, eficiencia y ambiente escolar) para establecer los planes de mejoramiento de su labor.

Los resultados internos de las pruebas SABER $3^{\circ}, 5^{\circ}$ y $9^{\circ}$ del año 2015 , muestran que los puntajes de los estudiantes del Norte de Santander se asemejan a los del resto del país, que oscilan entre niveles de desempeño insuficiente y mínimo. Aunque no se trate de una causa única (los problemas educativos por lo general son multidimensionales), es posible conjeturar que parte de este rendimiento deficitario de los estudiantes esté asociado a prácticas pedagógicas que no permiten entre ellos el desarrollo suficiente de las competencias específicas del área de Ciencias Naturales (ICFES, 2010).

Esta hipótesis surge, en buena medida, de la observación de un escenario frecuente en la educación básica en el que se combinan la falta de pertinencia y apropiación de los docentes por el conocimiento de las competencias específicas de su disciplina y la insuficiente motivación de los estudiantes en el desarrollo propio de sus competencias como estudiantes. Entre otros estudios confirmatorios de esta circunstancia puede citarse a Veloza (2015).

En relación con esta situación, los autores de este trabajo se han propuesto examinar y proponer estrategias para el fortalecimiento de la cultura ciudada- 
na, virtual, digital y democrática en ciencia, tecnología e innovación, mediante el empleo de la investigación como estrategia pedagógica apoyada en las TIC en instituciones educativas de Norte de Santander. Este artículo es un informe parcial de esta investigación referida a la descripción de la práctica pedagógica adoptada para el desarrollo de la competencia científica a partir del modelo pedagógico asumido en el municipio de Santiago, Norte de Santander, con base en las concepciones de los docentes sobre las competencias propias de la disciplina y el sistema de evaluación que se promueve en las instituciones educativas de la región. Concretamente, el propósito del trabajo se ha delimitado a la evaluación del nivel de desarrollo de las competencias pedagógicas que han alcanzado los docentes de la región en la enseñanza de la Ciencia.

De la observación de los contextos sociocultural y académico (principalmente del rendimiento deficitario de los estudiantes de octavo y noveno año) de la Institución Educativa Santiago Apóstol del municipio de Santiago, Departamento Norte de Santander, surgen los siguientes interrogantes de investigación: ¿Las prácticas pedagógicas del área de Ciencias Naturales favorecen el desarrollo de las competencias científicas o específicas de los estudiantes en los grados octavo y noveno de la institución educativa? ¿Qué opinión tienen los estudiantes acerca de las prácticas pedagógicas que son implementadas por el docente? ¿Qué estrategias pedagógicas verifican el nivel de desarrollo de competencias científicas en los estudiantes?

Como se ha mencionado, estos planteamientos parten de evaluaciones institucionales previas, que han mostrado los resultados bajos de las pruebas saber en el Área de Ciencias Naturales de los últimos años. En este sentido, un trabajo de investigación del tipo que los autores proponen contribuiría con la búsqueda de respuesta y la consecuente proposición de herramientas que contribuyan a superar tal situación en la enseñanza de las ciencias.

En efecto, según el Ministerio de Educación Nacional (a través de su Comité Ejecutivo) presentó un análisis en el año 2010 sobre los resultados de las Pruebas SABER de los grados 5 y 9. Según el Instituto Colombiano para la Evaluación de la Educación (en adelante ICFES, 2010):

En noveno grado, el 53\% de los estudiantes está en el nivel mínimo. Esta cifra muestra que un poco más de la mitad de los estudiantes al finalizar la básica secundaria reconoce algunas adaptaciones de los organismos al entorno, compara las propiedades de diversos materiales, identifica el estado físico de las sustancias a partir de la organización de sus partículas y reconoce qué preguntas pueden ser contestadas a partir de investigaciones científicas. Adicionalmente, presentan de forma apropiada sus resultados y procedimientos (p.16). 
Sin embargo, 17 de cada 100 estudiantes de este grado no demuestran las competencias mínimas exigidas en el área de Ciencias Naturales (ICFES, 2010, p. 16). De estos datos se desprende que el área de Ciencias Naturales necesita ser sometida a revisión a fin de mejorar los resultados académicos de las pruebas externas. El presente artículo intentará ofrecer información útil en relación con la identificación de las estrategias que promueven el desarrollo de competencias científicas en los jóvenes de estos niveles.

Entre los antecedentes internacionales conectados con la investigación se encuentra el trabajo de García et al. (2010) sobre innovación educativa y mejoramiento de las actitudes y competencias científicas de los estudiantes. La investigación consistió en la aplicación de un programa de innovación educativa para mejorar las actitudes hacia la metodología de los estudiantes de las asignaturas de las ciencias del comportamiento mediante el proceso de investigación. Los autores reportan que en el proyecto los estudiantes utilizaron datos reales para contrastar sus propias hipótesis utilizando diferentes análisis estadísticos y aplicando dos principios metodológicos claves para el contraste: el tamaño de la muestra y los enunciados ordinales.

Esta investigación de los estudiantes implicó el trabajo en grupo, el empleo de bases de datos en Internet para el desarrollo del marco teórico, delimitación de los objetivos del estudio, definición de variables, selección de estrategias para el análisis y discusión de los resultados. Cuando terminó la investigación de los estudiantes se valoraron el rendimiento académico y los cambios de actitudes hacia la metodología. Los resultados indicaron que 1) la realización de esta experiencia de investigación contrastada con datos reales mejoró la formación del estudiante y 2) supuso una toma de contacto con la realidad metodológica que favoreció sus actitudes.

Se espera que el manejo de estrategias metodológicas como las descritas en el trabajo anterior, permitan despertar el interés en los jóvenes por la investigación. Por ello, el aporte que se busca brindar mediante este trabajo se enmarca en las estrategias metodológicas que se proponen tanto en el campo del saber disciplinar como en la didáctica propia de las Ciencias Naturales.

En cuanto a investigaciones circunscritas al contexto colombiano, Restrepo (2007) indagó en las habilidades investigativas entre niños y niñas de 5 a 7 años estudiantes en instituciones oficiales y privadas de Manizales, Caldas. El trabajo tuvo como finalidad describir las habilidades investigativas (clasificación, planificación, formulación de hipótesis, experimentación y comprobación de hipótesis) en niños y niñas de 5 a 7 años, con el propósito de caracterizar dichas habilidades, establecer su nivel de desarrollo, las diferencias existentes entre los niños procedentes del sector oficial y privado y las posibles diferencias significativas entre géneros. 
Berrio y Torres (2009) se ocuparon de indagar sobre las concepciones de los docentes de Ciencias Naturales sobre competencias científicas y su desarrollo en las prácticas de aula. Entre los resultados, los autores destacan que cada docente es un sujeto que se ha constituido históricamente en un contexto sociocultural que ha posibilitado la construcción de determinadas concepciones sobre la ciencia, la enseñanza y el aprendizaje. Se trató, entonces, de abrir espacios de interacción entre docentes, que por una parte permitieran hacer visible dichas concepciones y, por otra, identificar otras opciones favorecedoras de los cambios que los mismos docentes decidan afrontar. Se logró caracterizar los saberes traducidos en actuaciones en las prácticas pedagógicas atendiendo a: 1) el saber disciplinar, 2) el saber enseñar el énfasis, 3) los criterios de organización y secuenciación de contenidos y 4) los criterios de selección y secuenciación de actividades de evaluación.

Tamayo (2014), por su parte, establece una discusión entre el objeto de estudio de la didáctica de las ciencias y su relación con el campo de la pedagogía, en la que se privilegia la tesis del desarrollo del pensamiento crítico de dominio específico. Para ello, presenta los resultados de investigación sobre la expresión del pensamiento crítico en niños y maestros de los grados cuarto y quinto de educación básica primaria de 56 instituciones públicas de Manizales, en el contexto de las Ciencias Naturales. En los resultados, el autor describe las concepciones de los maestros acerca del pensamiento crítico. El anterior proyecto demuestra el interés que se tiene por el desarrollo de competencias científicas, generadas desde la concepción misma de ciencia que tiene el maestro, desde la forma como concibe la importancia de la planeación, desarrollo y evaluación curricular. Este es, precisamente, el enfoque que se ha decidido adoptar en la presente investigación.

Finalmente, Hernández et al. (2017) buscaron analizar la percepción sobre la importancia y el desarrollo de competencias entre docentes que orientan el área de ciencias naturales, tanto en la formación y desempeño profesional como en su relación con el contexto educativo. Se realizó un estudio descriptivo con uso de metodología cuantitativa a través del cual se analizaron las percepciones de los docentes. Participaron en total ocho docentes de ciencias naturales que imparten las asignaturas de física y química en el nivel de educación media de dos instituciones educativas, una de carácter oficial y la otra privada, ambas en el municipio de Cúcuta (Colombia). Como instrumento se utilizó un cuestionario de escala Likert basado en las competencias genéricas del proyecto Tuning Latinoamérica.

Los resultados evidencian la existencia de discrepancias entre el imaginario de los actores en relación con las competencias y el grado de desarrollo que realmente alcanzan al interior de las instituciones. Se concluye que los docentes, con 
independencia del contexto educativo en el que laboran, valoran positivamente y otorgan mayor importancia a las competencias desarrolladas en el desempeño profesional que a las adquiridas durante la formación, especialmente las relacionadas con valores sociales y procesos de aprendizaje. Ello contrasta, por otra parte, con la escasa importancia que se concede a las competencias vinculadas con el contexto tecnológico internacional.

A fin de soportar teóricamente la investigación, se describen a continuación los principales referentes que se han abordado en ella:

\section{Concepción del área de Ciencias Naturales desde el Ministerio de Educación Nacional de Colombia}

Cuando el Ministerio de Educación Nacional establece la concepción de ciencias naturales en la Guía de Estándares Curriculares del área de Ciencias Naturales y Ciencias Sociales lo hace con suma cautela, pues "resulta riesgoso dar una definición consensuada sobre las ciencias naturales. Ellas son cuerpos de conocimientos que se ocupan de los procesos que tienen lugar en el mundo de la vida”. Se precisa que se trata de procesos naturales para referirse a todos aquellos procesos que, o bien no tienen que ver con el ser humano o, si lo tienen, es desde el punto de vista de especie biológica. El Ministerio de Educación Nacional (2014) también afirma que, desde una visión contemporánea de las ciencias y su enseñanza, existe la férrea convicción de que es necesario desarrollar las competencias de los estudiantes a partir de la conjugación de a) conceptos científicos, b) metodologías y maneras de proceder científicamente y c) compromiso social y personal en relación con el desarrollo científico y tecnológico.

Además, en la misma Guía, el Ministerio establece que en este eje de contenidos están contenidas las bases que permitirán a los educandos acercarse paulatina y rigurosamente al conocimiento y la actividad científica, de modo que, partiendo de la indagación, alcancen niveles de comprensión cada vez más complejos.

Por último, es importante asumir que la formación en ciencia requiere desarrollar en los estudiantes unas acciones concretas de pensamiento y producción, requeridas para alcanzar los estándares por conjunto de grados. Este proceso de formación debe partir de la comprensión del mundo y llegar hasta la aplicación de lo que los estudiantes aprenden, pasando por la investigación y la discusión sobre su importancia en el bienestar de las personas y el desarrollo de una sociedad democrática, justa, respetuosa y tolerante. 


\section{Del concepto de competencia a la competencia científica}

A menudo los profesores de ciencias cuya formación estuvo basada en otros modelos pedagógicos se preguntan en qué consiste la competencia científica (OCDE, 2016, p. 2). La respuesta que ofrece la OCDE es simple, pero a la vez compleja: "Saber pensar como un científico, saber hacer como un científico y, lo más importante, ser como un científico".

De acuerdo con Mantilla, Morales y Gómez (2011) se podría decir que una persona ha adquirido la competencia científica si es capaz de utilizar el conocimiento científico en contextos cotidianos, de aplicar los procesos que caracterizan las ciencias y sus métodos de investigación, al mismo tiempo que es consciente del papel que ejercen la ciencia y la tecnología en la sociedad tanto en la solución de problemas como en la génesis de nuevos interrogantes.

Es por ello por lo que las competencias científicas hacen referencia principalmente a la capacidad de entablar relación con las ciencias. Esta relación puede ser de dos tipos. La relación que tienen aquellos que por profesión están directamente comprometidos con la generación de conocimiento (científicos y académicos) y la que todo ciudadano puede establecer con ellas, aunque no de manera profesional. En este sentido, Hernández (2005) afirma que cuando se habla de competencias científicas se refiere a la capacidad de establecer un cierto tipo de relación con las ciencias, pero que la relación que los científicos de profesión tienen con las ciencias no es la misma que establecen con ellas quienes no están directamente comprometidos con la producción de los conocimientos sobre la naturaleza o la sociedad.

A este respecto Hernández (2005) señala sería deseable desarrollar en todos los ciudadanos, independientemente de la tarea social que les corresponde al menos un cierto grado de competencias científicas de modo que pueda relacionarse adecuadamente con el mundo contemporáneo, mediado cada vez más por la ciencia, pero principalmente por la tecnología. En el ambiente educativo conviene desarrollar las dos orientaciones de las competencias científicas (que de hecho no son para nada excluyentes), pero el segundo tipo de Competencias interesa especialmente a la educación básica y media porque tiene relación con la vida de todos los ciudadanos.

En este sentido, en la práctica pedagógica el maestro debe educar tanto desde la construcción del saber disciplinar y como de la didáctica propia de su disciplina, lo cual conlleva a un el proceso de enseñanza aprendizaje más efectivo.

Las prácticas pedagógicas y su aporte al proceso de enseñanza aprendizaje

Tal y como afirma Zuluaga (1999), 
La pedagogía no es solo un discurso acerca de la enseñanza, sino también una práctica cuyo campo de aplicación es el discurso. El maestro enfrenta sus conocimientos pedagógicos al discurso de las "teorías" o de las "ciencias" y el instrumento que utiliza para ellos es el método de enseñanza (p.10).

En este sentido, la práctica pedagógica consiste en una actividad social encomendada en gran parte al maestro, que debe adecuar el discurso de las ciencias a la edad de los sujetos que las aprenden de manera que les resulte asequible. Este discurso debe ser graduado a las formas de trabajo cotidiano en la enseñanza y, en este sentido, el maestro constituye una especie de bisagra que relaciona los discursos de las ciencias con el de su enseñanza. En el contexto de la enseñanza, el vínculo de los maestros con los conocimientos y los estudiantes se establece a través de la práctica pedagógica. La referencia a la adecuación del discurso de los manuales (que es como se refiere concretamente Zuluaga al discurso de la ciencia) tiene que ver con el hecho de en la mayor parte de las prácticas pedagógicas, los manuales de ciencias son el recurso didáctico más usado. Aun para la tecnología educativa, los manuales son los contenidos que se procesan en objetivos instruccionales, reduciendo la enseñanza de las ciencias a procesos de aprendizaje (Zuluaga, 1999).

En relación con lo anterior, en la actualidad hay sujeción a unos estándares de calidad que la temática relacionada con las competencias básicas de las diferentes áreas del saber atendiendo a la edad y el nivel escolar de los estudiantes. Cabe señalar que en la práctica pedagógica se deben emplear métodos y estrategias de aprendizaje. En este sentido, Palomares y Villareal (2009) afirman que la actual revolución informática requiere de un nuevo tipo de alfabetización vinculada al buen uso de las tecnologías y de la información, cuyo acceso no hace del sujeto un alfabetizado tecnológico. Lo relevante será el desarrollo de procesos formativos dirigidos a que cualquier sujeto aprenda a aprender (es decir, adquiera las habilidades para el aprendizaje autónomo a lo largo de su vida); sepa enfrentarse a la información (buscar, seleccionar, elaborar y difundir aquella información necesaria y útil); se cualifique laboralmente para el uso de las nuevas tecnologías de la información y comunicación y tome conciencia de las implicaciones económicas, ideológicas, políticas y culturales de la tecnología.

Otra de las prácticas pedagógicas que pueden generar competencias científicas a partir del método o estrategia de enseñanza se asocia a la enseñanza por resolución de problemas, pues constituye una estrategia que permite acercar a los estudiantes a una visión más contextualizada y menos deformada de ciencia, a la vez que contribuye al desarrollo de competencias científicas básicas (Palomares y Villareal, 2009). 
En el campo de la didáctica de las ciencias, la resolución de problemas ha sido estudiada como estrategia para generar cambios conceptuales, metodológicos y actitudinales, para superar la metodología del sentido común como capacidad relacionada con la estructuración de la información en la mente; o como proceso que puede enseñarse a los novatos a partir de la forma en que resuelven problemas los expertos, a través del diseño de herramientas heurísticas que guíen al estudiante en la resolución de problemas y, por último, como una estrategia para desarrollar la creatividad en los estudiantes (Palomares y Villareal, 2009).

En tal sentido, la importancia de realizar estos estudios sobre la enseñanza de las ciencias está enmarcada en el mejoramiento continuo que debe darse a las prácticas pedagógicas desde las políticas educativas vigentes, en el caso de Colombia las emitidas por el Ministerio de Educación Nacional, dirigidas a los maestros que orientan el área de ciencias naturales y educación ambiental y que requieren de un cambio o resignificación de los procesos de planeación, desarrollo y evaluación curricular, generando con ello un impacto positivo de motivación e interés de los estudiantes por el aprendizaje de las ciencias.

\section{Metodología}

Dado que se pretende evaluar el nivel de desarrollo de las competencias pedagógicas en el marco de la enseñanza de la ciencia a través de la exploración de las percepciones de los estudiantes sobre las competencias de sus docentes, se decidió establecer un análisis de sus relaciones desde un punto de vista cuantitativo y perspectiva descriptiva. Se procuró, en consecuencia, destacar las tendencias y presencia de las variables de interés en la población abordada a través de la observación, lo cual se cumplió mediante el uso de cuestionarios. Los datos obtenidos se procesaron aplicando estrategias de análisis descriptivo-bivariantes y multivariantes.

El nivel establecido para la investigación es de tipo descriptivo y puesto que se emplean técnicas multivariantes para reducir los datos a un conjunto de factores comunes, se considera un diseño multifactorial. Por una parte, se busca diagnosticar, a partir de la valoración de los estudiantes, cuáles son las competencias de planeación, desarrollo y evaluación que ponen en práctica los docentes en la enseñanza de la ciencia. Este reporte se basa en el uso de estadísticos descriptivos adecuados a la naturaleza de las variables analizadas. El estudio también consideró el uso de técnicas descriptivas multivariantes con la finalidad de establecer una taxonomía dentro del conjunto de estrategias que manejan los docentes. Por otra parte, en la investigación se incluyó un trabajo de campo que 
permitió describir las prácticas pedagógicas en el área de Ciencias Naturales que fomentan el desarrollo de competencias específicas o científicas propias del área de conocimiento.

El estudio siguió un enfoque secuencial. En este sentido, una vez que se precisó el objeto del estudio y el propósito de la investigación se siguieron las siguientes etapas: 1) Revisión de la literatura: búsqueda de antecedentes y modelos teóricos de referencia relacionados con las competencias pedagógicas para la enseñanza de la ciencia; 2) Selección de una muestra: delimitación del contexto de investigación, representado por estudiantes de los grados octavo y noveno de la Institución Educativa Santiago Apóstol, que alcanzan setenta estudiantes; 3) Diseño y validación de instrumentos: se revisó un conjunto de instrumentos para seleccionar los indicadores y confeccionar uno propio. El cuestionario diseñado fue validado por expertos a través de la técnica del juicio de expertos y se aplicaron previamente a una muestra piloto.

La etapa 4) fue la recolección de la información: los instrumentos fueron aplicados in situ mediante la modalidad cara a cara. Esta aplicación se hizo durante el horario de clases y bajo la supervisión de los investigadores. Los datos fueron registrados inicialmente en una hoja de cálculo en formato de Excel y luego se trasladaron al software para el análisis estadístico SPSS; 5) Análisis de la Información: se procesaron los datos a través del software SPSS, aplicando las técnicas de análisis estadístico adecuadas a la naturaleza de las variables. Los resultados fueron presentados en forma resumida con su respectiva interpretación; y 6) Conclusiones y hallazgos: en función de los resultados se destacan las principales líneas teóricas que rigen las acciones y se proponen las recomendaciones relacionadas con las competencias pedagógicas de los docentes de Educación Básica durante la enseñanza de la ciencia. En esta etapa final se hace referencia a algunas implicaciones teóricas y metodológicas del estudio.

A continuación, se detalla la secuencia:

La población objeto de estudio corresponde al alumnado de los grados octavo y noveno de la Institución Educativa Santiago Apóstol, que suman en total 70 estudiantes. Al considerarse todos los estudiantes para el estudio se establece una muestra censal. Para el desarrollo del proyecto se utilizó la encuesta. Su diseño siguió una metodología deductiva y estuvo fundamentada en la guía de evaluación docente del decreto 1278 (Ministerio de Educación Nacional, 2014), que consiste en la evaluación de tres tipos de competencias: disciplinares, pedagógicas y comportamentales. De esta tríada se escogieron solo las competencias pedagógicas, pues representan ellas solas una gran complejidad. Entre las competencias a evaluar se encuentran la planeación de la práctica educativa escolar, su desarrollo y el seguimiento y mejora de tal práctica considerando como indicadores el currículo, la didáctica y la metodología y, por último, la evaluación. 
Se seleccionaron los indicadores y se estableció como criterio de medición una escala tipo Likert de cinco puntos.

Para determinar la validez relacionada con el contenido, el instrumento fue sometido a un proceso de validación por juicio de expertos, a través de la cual se considera la opinión y las sugerencias de profesores universitarios especialistas en el área. A partir de sus observaciones se efectuaron las mejoras pertinentes. Adicionalmente, se diseñó una prueba piloto para analizar el comportamiento de cada ítem y calcular la confiabilidad de la escala. Una vez aplicado el instrumento a la muestra piloto de 30 sujetos y eliminadas o modificadas las preguntas sugeridas por el análisis de ítems y el juicio de expertos, se calculó nuevamente el estadístico Alpha de Cronbach, que arrojó un valor de 0,70, lo cual indica que el rango de confiabilidad de la escala aceptable (Pardo y Ruiz, 2002).

En consecuencia, se puede decir que la escala diseñada goza de credibilidad y consistencia interna, y que puede tomarse, además, como referente para futuras aplicaciones. En cuanto al propósito y contenido del instrumento, estuvo dirigido a los estudiantes a fin de que evaluaran a sus maestros del área de Ciencias Naturales desde los tres aspectos en que se desglosa la competencia pedagógica: la planeación curricular, el desarrollo curricular y el seguimiento y mejora (evaluación curricular).

Para la fase de análisis de los resultados, se eligió un software que permitió generar análisis cuantitativo, concretamente, el IBM SPSS Statistics, versión 24. En primer lugar, se reporta un análisis descriptivo con base en tablas de frecuencias conjuntas que muestran las tres dimensiones principales de las competencias pedagógicas. Luego, y con el fin de identificar variables subyacentes o factores que explicaran la configuración de las correlaciones dentro del conjunto de variables observadas, se realizó un análisis factorial exploratorio que permitió obtener una versión resumida de las competencias analizadas.

\section{Resultados}

Los resultados descriptivos se presentan en forma agrupada por tipos de competencia pedagógica: planeación, desarrollo y evaluación curricular. Seguidamente se hace una descripción del análisis factorial, reportando inicialmente una verificación de supuestos para la aplicación de esta técnica y se procede luego a la extracción e interpretación de los factores.

La tabla 1 muestra las distribuciones de frecuencia de los ítems 1 al 9, que corresponden al primer componente de la competencia pedagógica, denominado planeación curricular. Aquí se observa una clara tendencia hacia una ponderación de alta frecuencia en la valoración de los estudiantes en al menos cuatro de 
los ítems en esta escala: aplicación de la prueba diagnóstica al iniciar el periodo escolar $(92,6 \%)$, uso de distintos tipos de materiales para desarrollar las clases $(87,6 \%)$, aclarar dudas surgidas antes de las evaluaciones (81,3\%) y socialización y retroalimentación de las fallas de los estudiantes $(85,1 \%)$. En el otro extremo, con una baja representación, alrededor del $65 \%$ de los estudiantes afirma que nunca o casi nunca se fomentan actividades de trabajo individual y aproximadamente un $28 \%$ afirma que rara vez el docente se apoya en recursos tecnológicos o se vinculan los proyectos del área de ciencias con otras asignaturas.

Tabla 1. Competencias Pedagógicas: Planeación Curricular

\begin{tabular}{|c|c|c|c|c|c|c|}
\hline Indicador & & nunca & $\begin{array}{c}\text { casi } \\
\text { nunca }\end{array}$ & $\begin{array}{l}\text { algunas } \\
\text { veces }\end{array}$ & $\begin{array}{l}\text { casi } \\
\text { siem- } \\
\text { pre }\end{array}$ & $\begin{array}{l}\text { siem- } \\
\text { pre }\end{array}$ \\
\hline \multirow{2}{*}{$\begin{array}{l}\text { 1. Aplicación de prueba } \\
\text { diagnóstica }\end{array}$} & f & 0 & 3 & 3 & 17 & 57 \\
\hline & $\%$ & $0,0 \%$ & $3,8 \%$ & $3,8 \%$ & $21,3 \%$ & $71,3 \%$ \\
\hline \multirow{2}{*}{$\begin{array}{l}\text { 2. Uso de distintos tipos de } \\
\text { materiales }\end{array}$} & $f$ & 0 & 0 & 10 & 17 & 53 \\
\hline & $\%$ & $0,0 \%$ & $0,0 \%$ & $12,5 \%$ & $21,3 \%$ & $66,3 \%$ \\
\hline \multirow{2}{*}{$\begin{array}{l}\text { 3. Aplicación de ejemplos } \\
\text { relacionados con el entorno }\end{array}$} & $f$ & 1 & 0 & 23 & 31 & 25 \\
\hline & $\%$ & $1,3 \%$ & $0,0 \%$ & $28,8 \%$ & $38,8 \%$ & $31,3 \%$ \\
\hline \multirow{2}{*}{$\begin{array}{l}\text { 4. Desarrollo de proyectos } \\
\text { vinculados con otras materias }\end{array}$} & $f$ & 7 & 15 & 30 & 14 & 14 \\
\hline & $\%$ & $8,8 \%$ & $18,8 \%$ & $37,5 \%$ & $17,5 \%$ & $17,5 \%$ \\
\hline \multirow{2}{*}{$\begin{array}{l}\text { 5. Apoyo en recursos } \\
\text { tecnológicos }\end{array}$} & $f$ & 14 & 8 & 34 & 18 & 6 \\
\hline & $\%$ & $17,5 \%$ & $10,0 \%$ & $42,5 \%$ & $22,5 \%$ & $7,5 \%$ \\
\hline \multirow{2}{*}{$\begin{array}{l}\text { 6. Apoyo en publicaciones } \\
\text { científicas }\end{array}$} & f & 8 & 9 & 40 & 15 & 8 \\
\hline & $\%$ & $10,0 \%$ & $11,3 \%$ & $50,0 \%$ & $18,8 \%$ & $10,0 \%$ \\
\hline \multirow{2}{*}{$\begin{array}{l}\text { 7. Fomento del trabajo } \\
\text { individual }\end{array}$} & $f$ & 19 & 33 & 25 & 1 & 2 \\
\hline & $\%$ & $23,8 \%$ & $41,3 \%$ & $31,3 \%$ & $1,3 \%$ & $2,5 \%$ \\
\hline \multirow{2}{*}{$\begin{array}{l}\text { 8. Socialización y } \\
\text { retroalimentación de las fallas } \\
\text { de los estudiantes }\end{array}$} & $f$ & 0 & 4 & 16 & 19 & 41 \\
\hline & $\%$ & $0,0 \%$ & $5,0 \%$ & 20,0\% & $23,8 \%$ & $51,3 \%$ \\
\hline \multirow{2}{*}{$\begin{array}{l}\text { 9. Aclaratoria de dudas antes } \\
\text { de la evaluación }\end{array}$} & $f$ & 1 & 5 & 9 & 19 & 46 \\
\hline & $\%$ & $1,3 \%$ & $6,3 \%$ & $11,3 \%$ & $23,8 \%$ & $57,5 \%$ \\
\hline
\end{tabular}

Fuente: elaboración propia

En la tabla 2 se muestran las distribuciones de frecuencias de los ítems 10 al 19, 23 y 24, que se refieren al desarrollo de la práctica educativa escolar (desa- 
rrollo curricular). Como indicadores claves el 90\% de los estudiantes opina que sus docentes aclaran dudas cuando están trabajando en grupo, aproximadamente un $79 \%$ invita a los estudiantes a la reflexión y a realizar aportes para el desarrollo de los contenidos y un $75 \%$ de las opiniones ratifican que frecuentemente los docentes promueven la participación en el aula.

Tabla 2. Competencias Pedagógicas: Desarrollo Curricular

\begin{tabular}{|c|c|c|c|c|c|c|}
\hline Indicador & & nunca & $\begin{array}{c}\text { casi } \\
\text { nunca }\end{array}$ & $\begin{array}{l}\text { algunas } \\
\text { veces }\end{array}$ & $\begin{array}{c}\text { casi } \\
\text { siem- } \\
\text { pre }\end{array}$ & $\begin{array}{l}\text { siem- } \\
\text { pre }\end{array}$ \\
\hline \multirow{2}{*}{$\begin{array}{l}\text { 10. Presenta contenidos } \\
\text { desvinculados de la vida real }\end{array}$} & $\mathrm{f}$ & 14 & 12 & 28 & 14 & 12 \\
\hline & $\%$ & $17.5 \%$ & $15.0 \%$ & $35.0 \%$ & $17.5 \%$ & $15.0 \%$ \\
\hline \multirow{2}{*}{$\begin{array}{l}\text { 11. Relaciona los temas con } \\
\text { fenómenos actuales }\end{array}$} & $f$ & 1 & 5 & 31 & 30 & 13 \\
\hline & $\%$ & $1.3 \%$ & $6.3 \%$ & $38.8 \%$ & $37.5 \%$ & $16.3 \%$ \\
\hline \multirow[t]{2}{*}{ 12. Clase monótona y aburrida } & $f$ & 4 & 6 & 22 & 23 & 25 \\
\hline & $\%$ & $5.0 \%$ & $7.5 \%$ & $27.5 \%$ & $28.8 \%$ & $31.3 \%$ \\
\hline \multirow{2}{*}{$\begin{array}{l}\text { 13. Invita a la reflexión y a } \\
\text { realizar aportes }\end{array}$} & $f$ & 0 & 1 & 16 & 29 & 34 \\
\hline & $\%$ & $0.0 \%$ & $1.3 \%$ & $20.0 \%$ & $36.3 \%$ & $42.5 \%$ \\
\hline \multirow{2}{*}{$\begin{array}{l}\text { 14. Acompaña a los grupos } \\
\text { aclarando dudas }\end{array}$} & $\mathrm{f}$ & 0 & 0 & 8 & 16 & 56 \\
\hline & $\%$ & $0.0 \%$ & $0.0 \%$ & $10.0 \%$ & $20.0 \%$ & $70.0 \%$ \\
\hline \multirow[t]{2}{*}{ 15. Promueve la participación } & $f$ & 0 & 3 & 17 & 22 & 38 \\
\hline & $\%$ & $0.0 \%$ & $3.8 \%$ & $21.3 \%$ & $27.5 \%$ & $47.5 \%$ \\
\hline \multirow{2}{*}{$\begin{array}{l}\text { 16. Evalúa continuamente y } \\
\text { retroalimenta los resultados }\end{array}$} & $f$ & 2 & 2 & 32 & 29 & 15 \\
\hline & $\%$ & $2.5 \%$ & $2.5 \%$ & $40.0 \%$ & $36.3 \%$ & $18.8 \%$ \\
\hline \multirow[t]{2}{*}{ 17. Invita a la autoevaluación } & $f$ & 0 & 9 & 21 & 25 & 25 \\
\hline & $\%$ & $0.0 \%$ & $11.3 \%$ & $26.3 \%$ & $31.3 \%$ & $31.3 \%$ \\
\hline \multirow[t]{2}{*}{ 18. Promueve la co-evaluación } & $f$ & 3 & 10 & 33 & 19 & 15 \\
\hline & $\%$ & $3.8 \%$ & $12.5 \%$ & $41.3 \%$ & $23.8 \%$ & $18.8 \%$ \\
\hline \multirow{2}{*}{$\begin{array}{l}\text { 19. Retraso en la entrega de } \\
\text { evaluaciones }\end{array}$} & $f$ & 10 & 6 & 28 & 19 & 17 \\
\hline & $\%$ & $12.5 \%$ & $7.5 \%$ & $35.0 \%$ & $23.8 \%$ & $21.3 \%$ \\
\hline \multirow{2}{*}{$\begin{array}{l}\text { 23. Estrategias basadas en los } \\
\text { resultados de la evaluación }\end{array}$} & $f$ & 0 & 5 & 27 & 19 & 29 \\
\hline & $\%$ & $0.0 \%$ & $6.3 \%$ & $33.8 \%$ & $23.8 \%$ & $36.3 \%$ \\
\hline \multirow{2}{*}{$\begin{array}{l}\text { 24. Dificultad para explicar en } \\
\text { función del tiempo estipulado }\end{array}$} & $f$ & 6 & 6 & 12 & 15 & 41 \\
\hline & & $7.5 \%$ & $7.5 \%$ & $15.0 \%$ & $18.8 \%$ & $51.3 \%$ \\
\hline
\end{tabular}

Fuente: elaboración propia 
Por otra parte, el 65\% de los estudiantes destacan que sus docentes presentan contenidos desvinculados de la vida real, un $45 \%$ cuestiona la retroalimentación de los resultados de la evaluación y un 16\% manifiesta que se promueve muy poco la co-evaluación. A pesar de que en el uso de estrategias con base en resultados de la Evaluación tiene una valoración positiva en al menos un 60\% del grupo, un 55\% de los estudiantes cuestionan la puntualidad en la entrega de los resultados de la evaluación.

Tabla 3. Competencias Pedagógicas: Seguimiento y Mejora (Evaluación Curricular)

\begin{tabular}{|c|c|c|c|c|c|c|}
\hline Indicador & & nunca & $\begin{array}{c}\text { casi } \\
\text { nunca }\end{array}$ & $\begin{array}{c}\text { algunas } \\
\text { veces }\end{array}$ & $\begin{array}{c}\text { casi } \\
\text { siem- } \\
\text { pre }\end{array}$ & $\begin{array}{l}\text { siem- } \\
\text { pre }\end{array}$ \\
\hline \multirow{2}{*}{$\begin{array}{l}\text { 20. Desarrollo de contenidos } \\
\text { de acuerdo con lo programado }\end{array}$} & $f$ & 0 & 1 & 7 & 27 & 45 \\
\hline & $\%$ & $0.0 \%$ & $1.3 \%$ & $8.8 \%$ & $33.8 \%$ & $56.3 \%$ \\
\hline \multirow[t]{2}{*}{ 21. Impone el criterio propio } & $\mathrm{F}$ & 17 & 15 & 22 & 15 & 11 \\
\hline & & $21.3 \%$ & $18.8 \%$ & $27.5 \%$ & $18.8 \%$ & $13.8 \%$ \\
\hline \multirow{2}{*}{$\begin{array}{l}\text { 22. Uso de diferentes medios } \\
\text { e instrumentos de evaluación }\end{array}$} & $\mathrm{F}$ & 1 & 6 & 24 & 26 & 23 \\
\hline & $\%$ & $1.3 \%$ & $7.5 \%$ & $30.0 \%$ & $32.5 \%$ & $28.8 \%$ \\
\hline \multirow{2}{*}{$\begin{array}{l}\text { 25. Relaciona las practicas con } \\
\text { lo visto en el aula }\end{array}$} & $\mathrm{F}$ & 6 & 9 & 13 & 14 & 38 \\
\hline & $\%$ & $7.5 \%$ & $11.3 \%$ & $16.3 \%$ & $17.5 \%$ & $47.5 \%$ \\
\hline \multirow{2}{*}{$\begin{array}{l}\text { 26. Conecta el aprendizaje } \\
\text { para comprender fenómenos }\end{array}$} & $\mathrm{F}$ & 0 & 1 & 9 & 19 & 51 \\
\hline & & $0.0 \%$ & $1.3 \%$ & $11.3 \%$ & $23.8 \%$ & $63.8 \%$ \\
\hline
\end{tabular}

Fuente: elaboración propia

La tabla 3, recoge los resultados de los ítems 20, 21 y 22 y los ítems 25 y 26, que hacen referencia al seguimiento y mejora de la práctica educativa escolar, lo que comúnmente se conoce como evaluación curricular. Aproximadamente el $90 \%$ de los estudiantes opina que sus docentes frecuentemente desarrollan los contenidos de acuerdo con lo programado y que promueven el aprendizaje de la ciencia para comprender los fenómenos reales. Aproximadamente un 70\% del grupo no está de acuerdo en señalar al docente como impositivo a la hora de establecer criterios y un $65 \%$ opina que siempre trabaja con la finalidad de relacionar el trabajo del laboratorio con lo visto en el aula. Por otra parte, cerca del $40 \%$ opina que regularmente se adoptan distintos medios e instrumentos de evaluación. 
Como una segunda aproximación a los datos y con el fin de reducir la dimensionalidad de los indicadores aplicados, es decir, con la finalidad de encontrar grupos de ítems a través de sus correlaciones para identificar factores o categorías comunes, se aplicó un Análisis Factorial (AF) de carácter exploratorio. La aplicación de esta técnica multivariante supone al menos la consideración de tres supuestos (Pardo y Ruiz, 2002): 1) La determinación del índice de adecuación muestral (KMO); 2) Un valor del determinante de la matriz de correlaciones distinto a cero y 3) la prueba de esfericidad de Barlett, que demuestra que la matriz de correlaciones es distinta a la matriz identidad. El valor del KMO alcanzó el valor de 0,65, lo cual resulta un valor mínimo de adecuación de la muestra para proceder con el análisis; el valor del determinante es 0,017 , distinto de cero, y la prueba de Barlett arroja un valor de $\chi^{2}=653,23$ con $\mathrm{gl}=325 \mathrm{y}$ un p-valor asociado de $\mathrm{p}=0,00<0,05$, lo que conduce a rechazar la hipótesis nula de que la matriz de correlaciones es la matriz identidad. Por otra parte, una inspección visual de las correlaciones indica una gran mayoría con valores superior a 0,30, lo que ratifica la conveniencia de aplicar el AF (Hair, Anderson, Tatham, y Black, 2002).

Luego de evaluados los supuestos básicos, se procedió a correr el AF, adoptando como método de extracción de factores los componentes principales (Peña, 2002), que constituyen el método por defecto proporcionado por el software SPSS. Basado en la revisión de los fundamentos teóricos acerca de la práctica pedagógica y el fortalecimiento de las competencias científicas a través de la revisión de la literatura y los lineamientos y estándares curriculares del Ministerio de Educación Nacional de Colombia, se fijó en cinco el número óptimo de factores. La composición del porcentaje de varianza explicada por factor se muestra en la tabla 5.

Tabla 5. Varianza total explicada

\begin{tabular}{cccccccc}
$\begin{array}{c}\text { Compo- } \\
\text { nente }\end{array}$ & Autovalores iniciales & $\begin{array}{c}\text { Sumas de las saturaciones al } \\
\text { cuadrado de la extracción }\end{array}$ & & $\begin{array}{c}\text { Suma } \\
\text { de las } \\
\text { satura- } \\
\text { ciones al } \\
\text { cuadrado } \\
\text { de la ro- } \\
\text { tación }\end{array}$ \\
\hline 1 & Total & $\begin{array}{c}\text { \% de la } \\
\text { varianza }\end{array}$ & $\begin{array}{c}\text { \% acu- } \\
\text { mulado }\end{array}$ & Total & $\begin{array}{c}\text { \% de la } \\
\text { varianza }\end{array}$ & $\begin{array}{c}\text { \% acu- } \\
\text { mulado }\end{array}$ & Total \\
\hline 2 & 5,391 & 20,733 & 20,733 & 5,391 & 20,733 & 20,733 & 4,035 \\
\hline & 2,180 & 8,386 & 29,120 & 2,180 & 8,386 & 29,120 & 3,105 \\
\hline
\end{tabular}




\begin{tabular}{cccccccc}
\hline 3 & 1,684 & 6,475 & 35,595 & 1,684 & 6,475 & 35,595 & 3,776 \\
\hline 4 & 1,563 & 6,012 & 41,607 & 1,563 & 6,012 & 41,607 & 2,125 \\
\hline 5 & 1,499 & 5,767 & 47,374 & 1,499 & 5,767 & 47,374 & 1,880 \\
\hline 6 & 1,345 & 5,173 & 52,547 & & & & \\
\hline 7 & 1,300 & 4,999 & 57,545 & & & & \\
\hline 8 & 1,218 & 4,685 & 62,231 & & & & \\
\hline$\ldots$ & $\ldots$ & $\ldots$ & $\ldots$ & & & & \\
\hline 26 &, 137 &, 526 & 100,000 & & & &
\end{tabular}

Source: Self-compiled by researcher

Los cinco factores que se describen en la tabla explican aproximadamente el $47 \%$ de la variabilidad del conjunto de datos, lo cual se considera óptimo (Hair, Anderson, Tatham y Black, 2002). El factor 1, el principal y el que mayor aporta a la explicación de la varianza, representa el 20,7\% de la solución. El segundo factor explica aproximadamente el $8 \%$. Los restantes tres factores explican entre el $6 \%$ y el $5 \%$ de la solución. Con estos cinco factores se logra reducir significativamente el número de variables originales, que era de veintiséis.

Tabla 6. Estructura factorial rotada

\begin{tabular}{lccccc} 
& \multicolumn{5}{c}{ Componente } \\
\cline { 2 - 6 } & 1 & 2 & 3 & 4 & 5 \\
\hline 9. Aclara dudas antes de la evaluación &, 772 &, 327 &, 303 & 189 &, 288 \\
\hline 14. Acompaña a los grupos aclarando dudas &, 759 &, 251 &, 292 &, 097 &, 018 \\
\hline 15. Promueve la participación &, 744 &, 056 &, 270 &, 106 & 118 \\
\hline 8. Socialización y retroalimenta fallas & 571 &, 050 &, 477 & 406 &, 382 \\
\hline 2. Usa distintos tipos de materiales &, 389 &, 343 &, 285 &, 148 & -226 \\
\hline 10. Presenta temas desvinculados de la vida real &,- 298 &,- 022 & -152 &,- 285 &,- 005 \\
\hline 7. Fomenta el trabajo individual &, 011 &,- 628 &,- 126 &, 114 &, 132 \\
\hline 22. Usa diferentes medios de evaluación &, 245 & 586 &, 288 & 441 &,- 089 \\
\hline 26. Conecta el aprendizaje con problemas reales &, 177 & 515 &, 262 & 110 &, 006 \\
\hline 13. Invita a la reflexión y a realizar aportes &, 503 &, 510 &, 365 &, 299 &, 026 \\
\hline 4. Realiza proyectos vinculados con otras materias & 330 &, 509 &, 128 &,- 129 &, 318 \\
\hline 11. Relaciona los temas con fenómenos actuales & 164 &, 472 &, 033 &, 027 &, 195 \\
\hline 18. Promueve la co-evaluación & 366 & 461 &, 398 &,- 314 & -115 \\
\hline
\end{tabular}




\begin{tabular}{lccccc}
\hline 1. Aplica pruebas diagnosticas & ,304 & .381 & 362 &,- 007 &,- 279 \\
\hline 23. Planifica estrategias con base en resultados & 529 &, 327 & 736 & 157 &,- 060 \\
\hline 12. Clase monótona y aburrida &, 257 &,- 125 &, 647 &, 394 & 450 \\
\hline 25. Relación de la práctica con lo visto en el aula & 519 &, 097 & 626 &, 312 &, 059 \\
\hline 3. Aplica ejemplos relacionados con el entorno & -116 &, 294 & 605 &, 016 &, 005 \\
\hline 16. Evalúa continuamente y retroalimenta & 331 & 069 & 580 &, 078 &, 000 \\
\hline 17. Invita a la autoevaluación &, 340 & 410 &, 510 &,- 087 &,- 209 \\
\hline 24. Dificultad para explicar en función del tiempo &, 280 &,- 322 &, 065 & 661 &, 312 \\
\hline 20. Desarrolla contenidos en función de lo planeado &, 227 &, 060 &, 134 &, 516 &,- 019 \\
\hline 21. Impone el criterio propio &, 139 &,- 238 &,- 161 &,- 513 &, 054 \\
\hline 19. Retraso en la entrega de las evaluaciones &, 124 &,- 167 &, 000 &, 043 & 668 \\
\hline 6. Apoyo en publicaciones científicas &, 121 &, 200 &, 062 &, 008 &, 542 \\
\hline 5. Apoyo en recursos tecnológicos &,- 059 &, 291 &, 470 & 341 &, 486
\end{tabular}

Source: Self-compiled by researcher

Seguidamente, se realizó la rotación de factores: "partiendo del hecho de que los factores obtenidos pueden estar correlacionados entre ellos, se optó por un método de rotación oblicua" (Pardo y Ruiz, 2002) para así obtener una solución rotada que facilitó la configuración y posterior interpretación de los factores. En la tabla 6 se evidencia la distribución de las variables observadas en la reducción a cinco factores.

Las cargas factoriales representan el peso de cada una de las variables en los factores obtenidos en la solución factorial. Se considera que la variable observada satura mejor en el factor cuya carga factorial es más alta. La tabla 6 muestra las cargas factoriales de cada variable observada en cada uno de los factores obtenidos y se resalta el mayor peso factorial en el factor donde satura la variable. Esta tabla nos facilita la obtención de la estructura factorial en función de las variables observadas.

Las cargas factoriales obtenidas oscilan en valor absoluto entre 0,48 y 0,77 , lo cual da cuenta del peso significativo que representa cada variable en el factor donde mejor satura. El primer factor, que explica el $20,73 \%$ de la varianza del modelo factorial presentado, está compuesto por los ítems 2, 8, 9, 10, 14 y 15 . El segundo factor agrupa ocho ítems: 1, 4, 7, 11, 13, 18, 22 y 26, y representa el 8,38\% de la solución. El factor tres está conformado por los ítems 3, 12, 16, 17,23 y 25 , y representa el 6,4\%. El cuarto factor abarca los ítems 20,21 y 24 , y explican el 6\% de la solución. Los ítems 5, 6 y 19 forman parte del factor 5, y representan el 5,7\% de la explicación de la solución. 
A partir de este proceso se consigue denominar cada uno de los factores con el fin de atribuir el significado a los hallazgos encontrados. "El proceso implica la interpretación sustantiva del patrón de cargas factoriales para las variables, incluidos sus signos, en un esfuerzo por denominar cada uno de los factores" (Hair, Anderson, Tatham y Black, 2002). Durante este proceso se consideraron todas las variables que conforman el factor y al mismo tiempo el peso que representa cada una en su interior, es decir, su carga factorial.

El Factor I se denomina retroalimentación. En él se describen las acciones que se desarrollan en las prácticas pedagógicas bajo la responsabilidad del docente en el área de Ciencias Naturales. Los estudiantes afirman que, desde su perspectiva, los docentes aclaran las dudas e inquietudes que se presentan en las clases y que hacen acompañamiento a los trabajos que se realizan en grupo; asimismo, utilizan diferentes tipos de materiales para el desarrollo de la clase, durante cuyo desarrollo promueven la participación de los estudiantes e involucran mecanismos de socialización de fallas en las evaluaciones, aunque en ocasiones desvinculan los temas de la realidad.

El Factor II, denominado estrategias de evaluación, describe acciones de los docentes para el cumplimiento de evaluaciones realizadas como mecanismo de seguimiento y control de los procesos desarrollados en las prácticas pedagógicas. En este sentido, los estudiantes afirman que sus docentes aplican siempre pruebas diagnósticas y que realizan procesos de co-evaluación y talleres que invitan a la reflexión y a la producción de aportes relacionando temáticas de actualidad que los motive al trabajo individual, la resolución de problemas y la realización de proyectos con otras materias.

El Factor III se denomina estrategias didácticas y de acuerdo con él, los estudiantes estiman que el docente realiza sus clases en función de lo planificado y relaciona el desarrollo de los temas con el entorno, utiliza diversas estrategias y tiene en cuenta los resultados de las evaluaciones, manteniendo siempre una evaluación permanente y continua sobre la base de los contenidos trabajados en clase; asimismo, orienta la autoevaluación, aunque en ocasiones, siempre de acuerdo con la opinión de los encuestados, la clase se muestra monótona y aburrida.

El desarrollo de contenidos constituye el Factor IV. De acuerdo con los estudiantes, el docente desarrolla el contenido teniendo en cuenta solo su criterio. También trata de abarcar todos los contenidos programados, aunque demuestra dificultad al explicar algunas temáticas.

Para finalizar, el factor $\mathrm{V}$ se denominó recursos para la enseñanza. Aquí los estudiantes afirman que los docentes hacen uso de diversas estrategias y recursos tecnológicos con el fin de innovar: internet, computadores, tabletas, video beam y otros medios a través de los cuales procura motivar las prácticas pedagó- 
gicas y promocionar la investigación para facilitar la comprensión de las temáticas; sin embargo, esto conlleva en ocasiones al retraso en la entrega de las notas.

Los hallazgos de la investigación permiten orientar, por una parte, una descripción de las estrategias utilizadas por los docentes de ciencias en su praxis pedagógica desde la óptica de los estudiantes, y por otra, una identificación de las estrategias que promueven el desarrollo de competencias científicas. En forma global, se devela la utilización de recursos didácticos y prácticas de laboratorio. Sin embargo, estas acciones no siempre están articuladas con las necesidades de los estudiantes, pues ellos mismos expresan que en ocasiones las clases se presentan descontextualizadas de la realidad y se tornan monótonas y aburridas.

Los resultados descriptivos resaltan que los docentes ven la necesidad de aplicar las pruebas diagnósticas como base de la planeación curricular. Por su intermedio, además, se seleccionan los materiales a utilizar en la aplicación de las secuencias didácticas, cumpliendo así con las funciones básicas de la evaluación para determinar la situación de cada alumno en el aula (Jorba y Sanmartí, 1993).

Por otra parte, queda en evidencia que, aunque los docentes llevan a sus clases temas de actualidad, existen otros aspectos que también deben involucrarse en la didáctica de las ciencias para promover motivación e interés por las clases. En este sentido, se debe reforzar el trabajo de epistemología y didáctica propia de la disciplina, tal como lo establece el Ministerio de Educación Nacional en los Estándares Básicos (2004):

La Didáctica de las Ciencias se ha desarrollado vertiginosamente y comienza a contemplarse en la actualidad como disciplina con cuerpo teórico propio que al mismo tiempo debe favorecer en los estudiantes la fundamentación conceptual, actitudinal y metodológica necesarias que dé cuenta de la problemática asociada a la enseñanza y al aprendizaje de las ciencias (p. 43).

De lo anterior se desprende que cuando en las prácticas pedagógicas se adopta una didáctica especializada en la enseñanza de las ciencias, se acerca al estudiante a la realidad de las acciones que permiten comprender los fenómenos científicos, lo cual constituye una de las propuestas que fundamentan los estándares de calidad de Ciencias Naturales y que se debe implementar en el desarrollo curricular.

Los resultados también permitieron destacar que el trabajo de seguimiento y evaluación curricular está direccionado únicamente por los docentes, sin participación alguna los estudiantes. También mostraron que se requiere más trabajo práctico como proceso de evaluación y que la resolución de problemas de contexto, y que ambos deben ser cada vez más amplios y, fundamentalmente, que se incluyan en ese plan de mejoramiento los aportes dados por los estu- 
diantes. Sobre este particular, es importante recordar que, para el Ministerio de Educación Nacional de Colombia, en el documento de los Estándares Básicos de Competencias en Ciencias Sociales y Ciencias Naturales (2004) establece que

Si la ciencia está formada por un conjunto de saberes que están en permanente cambio, en donde la revisión y el análisis crítico de lo que se hace es fundamental, la evaluación es un componente que no puede faltar en la formación en ciencias. Una evaluación que sea vista, al igual que la ciencia, como proceso, es decir que sea permanente, que arroje luces sobre el camino recorrido y el que se seguirá recorriendo (p.112).

Lo anterior guarda consonancia con los resultados de la encuesta aplicada a los estudiantes, pues "ya no es posible una evaluación dirigida a detectar errores, puntos de quiebre. Se trata de una evaluación orientada a identificar fortalezas que permitan superar las debilidades, una evaluación para determinar qué están aprendiendo realmente los y las estudiantes" (MEN, 2004: 112). Tal y como lo expresan los resultados, se debe dar uso de diferentes instrumentos de evaluación y, además, de manera permanente.

\section{Conclusiones}

Una de las conclusiones que derivan de este estudio es que los estándares curriculares emanados del MEN destacan que el proceso de evaluación ha de identificar las fortalezas del estudiante para superar las dificultades. Estos lineamientos hacen énfasis en que los docentes deben buscar las herramientas adecuadas para garantizar el proceso de enseñanza aprendizaje. Por otra parte, la guía metodológica 31 emanada por el Ministerio de Educación Nacional de Colombia (2008), establece los parámetros de Evaluación Anual de Desempeño Laboral y en él se describen los criterios que debe tener un docente para evidenciar el trabajo de organización escolar destacando las funciones de planeación, ejecución y evaluación de las diferentes actividades curriculares en función de los proyectos institucionales y considerando el contexto de los estudiantes

La organización de los ítems en factores permite valorar que la acción del docente está más concentrada en los aspectos didácticos y no una verdadera formación científica de sus estudiantes, lo que evidencia que hace falta conformar comunidades científicas que permitan el intercambio de estrategias, métodos y producto de investigaciones para fortalecer el desarrollo de competencias científicas propias del área según el grado de escolaridad en el que se encuentra el 
estudiante. Según los Estándares Básicos de Competencia en Ciencias Naturales (2004),

para poder fortalecer estos aprendizajes en los estudiantes es necesario que también el maestro se involucre en procesos de conformación de comunidad científica y así, de manera conjunta con otros y otras docentes, comparta sus experiencias, debata sus posturas, profundice sus conocimientos -tanto de su disciplina como de otras- y evalúe sus actuaciones de enseñanza. Estas redes de colaboración entre docentes pueden ser de gran valor para mantener posturas críticas y reflexivas sobre la propia práctica e introducir modificaciones a la práctica pedagógica (p.11).

En cuanto a las competencias científicas, se hace necesaria la profundización en su desarrollo entre los estudiantes de Educación Básica, sobre todo en el uso del conocimiento científico, la indagación y la explicación de fenómenos. El bajo desarrollo de competencias durante la práctica pedagógica de los maestros se explica desde los planteamientos de Furió y Gil (1994) sobre los problemas para la enseñanza de las ciencias: El primero, tiene que ver con las preconcepciones del propio profesorado, tanto en lo que se refiere a los temas científicos (Carrascosa y Gil, 1985) como, sobre todo, en lo que toca a la manera de enseñar (Bromme, 1988; Hewson y Hewson, 1987). El segundo problema que destaca es la familiarización de los estudiantes con las características del trabajo científico.

Esta reflexión epistemológica es necesaria no solo para favorecer una orientación más creativa de los trabajos prácticos sino para hacer ver el profundo cambio epistemológico y metodológico asociado al surgimiento de la ciencia moderna y al aprendizaje de los conocimientos científicos. El tema de las preconcepciones de sentido común y del 'cambio conceptual' converge así con el de la familiarización con la metodología científica y el 'cambio metodológico' (Gil y Carrascosa, 1985).

Los planteamientos anteriores reafirman la importancia de abordar desde concepciones epistemológicas apropiadas la planeación y el desarrollo curricular. En este sentido, se hace indispensable que en las instituciones de Educación Básica los maestros de la disciplina profundicen más en los fundamentos epistemológicos y didácticos que permitan la adecuada promoción y desarrollo de las competencias específicas de las ciencias naturales.

En esta dirección, los Estándares Básicos de Competencia en Ciencias Naturales (2004), en cuanto política educativa nacional, han establecido que lo que se busca es que 
estudiantes, maestros y maestras se acerquen al estudio de las ciencias como científicos y como investigadores, pues todo científico - grande o chico- se aproxima al conocimiento de una manera similar, partiendo de preguntas, conjeturas o hipótesis que inicialmente surgen de su curiosidad ante la observación del entorno y de su capacidad para analizar lo que observa (p.8).

Desde esta perspectiva, la política educativa nacional estableció la necesidad de acercar a los maestros y estudiantes al estudio de la ciencia como científicos, lo cual solo es posible cuando el maestro orienta prácticas pedagógicas desde la didáctica propia de la disciplina.

En cuanto a la inclusión de las TIC, su uso es desde hace tiempo imprescindible en la planeación, desarrollo y evaluación curricular, pues con su auxilio se fortalecen las prácticas pedagógicas y, en consecuencia, el proceso de aprendizaje. Estas consideraciones han sido revalidadas durante esta investigación.

El desarrollo de esta investigación, finalmente, permitió generar una reflexión de la práctica pedagógica en torno al componente pedagógico: planeación, desarrollo y evaluación curricular, desde la perspectiva del estudiante como un actor pedagógico participante de su proceso de formación; además, el proceso, generó unos factores bastantes críticos que se deben tener presente en los criterios que actualmente maneja el Ministerio de Educación Nacional Colombiano en la evaluación de la competencia docente: contexto escolar, planeación curricular, praxis pedagógica y ambiente en el aula.

\section{Referencias}

Berrio, A. y Torres, M. (2009). Concepciones de los docentes de ciencias naturales sobre competencias científicas y su desarrollo en las prácticas de aula (Tesis de Maestría). Montería: Universidad de Córdoba, Colombia.

Bromme, R. (1988). Conocimientos profesionales de los profesores. Enseñanza de las Ciencias, 6(1), 19-29.

Carrascosa, J. y Gil, D. (1985). La metodología de la superficialidad y el aprendizaje de las ciencias. Enseñanza de las Ciencias (3), 113-120.

Furió, C. (1994). Tendencias actuales en la formación del profesorado de ciencias. Enseñanza de las Ciencias, 12(2), 188-199. 
Furió, C. y Gil, D. (1989). La didáctica de las ciencias en la formación inicial del profesorado: una orientación y un programa teóricamente fundamentados. Enseñanza de las Ciencias, 7(3), 257-265.

García, J. F., Gracia, E., Fuentes, M., Lila, M., y Pascual, J. (2010). La innovación educativa desde la metodología: Mejora de las actitudes y competencias científicas de los alumnos. Escritos de Psicología, 3(4), 1-10.

Hair, J. F., Anderson, R., Tatham, R. y Black, W. (2002). Análisis multivariante. Prentice Hall Iberia: Madrid.

Hernández, C. (2005). Foro educativo nacional. Qué son las competencias científicas: Colombia Aprende. Recuperado de http://www.colombiaaprende.edu.co/ html/docentes/1596/articles-89416_archivo_5.pdf

Hernández, C., Pabón, C. y Prada, R. (2017). Desarrollo de competencias y su relación con el contexto educativo entre docentes de ciencias naturales. Revista Virtual Universidad Católica del Norte, (51), 194-215. Recuperado de http://revistavirtual.ucn.edu.co/index.php/RevistaUCN/article/ view/852/1370

Hewson, P. W. y Hewson, M. (1987). Science teachers' conception of teaching: Implications for teacher education. International Journal of Science Education, 9(4), 425-440.

ICFES (2010). Mejor saber. Saber $5^{\circ}$ y $9^{\circ} 2009$ resultados nacionales. Resumen ejecutivo. Recuperado de http://www.icfes.gov.co/docman/investigadores-y-estudiantes-de-posgrado/informes-de-resultados-evaluaciones-nacionales / informes-resultados-saber-3-5-y-9/ 102 1-saber-5o-y-9o-2009-resultados-nacionales-resumen-ejecutivo/file? force-download $=1$

ICFES (2013). Colombia en pisa 2012 Informe nacional de resultados. Resumen ejecutivo. Recuperado de: http://www.icfes.gov.co/docman/instituciones-educativas-y-secretarias/evaluaciones-internacionales-investigadores/pisa/pisa-2012/2703-resumen-ejecutivo-informe-nacional-de-resultados-colombia-en-pisa-2012/file? force-download = 1

Jorba, J. y Sanmartí, N. (1993). La función pedagógica de la evaluación. Aula de innovación educativa (20), 20-30. 
Mantilla, D., Morales. A, y Gómez L. C. (2011). Diseño de un sistema de formación de competencias, a propósito de la apropiación de la noción de propiedad intelectual, apoyado en tecnología de la información. Zona Próxima (15), 22-39.

Ministerio de Educación Nacional de Colombia. (2004). Estándares básicos de competencias en ciencias sociales y ciencias naturales. La formación en ciencias jel desafio!. Recuperado de http://www.mineducacion.gov.co/1621/articles-116042_archivo_pdf3.pdf

Ministerio de Educación Nacional de Colombia. (2008). Guía Metodológica $N^{\circ}$ 31. Evaluación Anual de Desempeño Laboral. Docentes y Directivos Docentes del Estatuto de Profesionalización Docente Decreto Ley 1278 de 2002. Recuperado de http://www.mineducacion.gov.co/1621/articles-169241_archivo_pdf. pdf

Ministerio de Educación Nacional de Colombia. (2014). Evaluación de competencias para el ascenso o reubicación de nivel salarial en el Escalafón de Profesionalización Docente de los docentes y directivos docentes regidos por el Decreto Ley 1278 de 2002. Docente de básica primaria. Documento guía. Bogotá: MEN

Organización para la Cooperación y el Desarrollo Económicos, OCDE. (2016). PISA 2015. Resultados clave. Recuperado de https://www.oecd.org/pisa/ pisa-2015-results-in-focus-ESP.pdf

Palomares, A. L. y Villareal, M. E. (2009). Material educativo computacional para el desarrollo de competencias científicas. Studiositas, 4(1), 17-26.

Pardo M., A y Ruiz, M. A. (2002). SPSS 11 Guía para el análisis de datos. Madrid: Editorial McGraw Hill.

Peña, D. (2002). Análisis de Datos Multivariantes. Madrid: Editorial McGrawHill.

Restrepo, F. (2007). Habilidades investigativas en niños y niñas de 5 a 7 años de instituciones oficiales y privadas de la ciudad de Manizales (Tesis de doctorado). Universidad de Manizales-Centro de Estudios Avanzados en Niñez y Juventud.

Tamayo, O. E. (2014). Pensamiento crítico dominio-específico en la didáctica de las ciencias. TED: Tecné, Episteme y Didaxis (2), 25-46. 
Veloza, R. A. (2015). Prácticas pedagógicas que desarrollan competencias científicas en estudiantes de sexto y séptimo grado de las instituciones educativas Centro Educativo Rural Florentino Blanco e Institución Educativa Santiago Apóstol (Tesis de Maestría). Universidad Francisco de Paula Santander, Cúcuta.

Zuluaga, O. (1999). Pedagogía e Historia. Santafé de Bogotá: Siglo del hombre. Editores. 